Honam Mathematical J. 34 (2012), No. 1, pp. 019-034

http://dx.doi.org/10.5831/HMJ.2012.34.1.19

\title{
A FIXED POINT APPROACH TO THE STABILITY OF THE MIXED TYPE FUNCTIONAL EQUATION
}

\author{
Sun SoOK Jin AND YANG-Hi LeE
}

\begin{abstract}
In this paper, we investigate the stability of a functional equation

$f(x+y+z)-f(x+y)-f(y+z)-f(x+z)+f(x)+f(y)+f(z)=0$ by using the fixed point theory in the sense of L. Cădariu and V. Radu.
\end{abstract}

\section{Introduction}

In 1940, S. M. Ulam [19] raised a question concerning the stability of homomorphisms: Given a group $G_{1}$, a metric group $G_{2}$ with the metric $d(\cdot, \cdot)$, and a positive number $\varepsilon$, does there exist a $\delta>0$ such that if a mapping $f: G_{1} \rightarrow G_{2}$ satisfies the inequality

$$
d(f(x y), f(x) f(y))<\delta
$$

for all $x, y \in G_{1}$ then there exists a homomorphism $F: G_{1} \rightarrow G_{2}$ with

$$
d(f(x), F(x))<\varepsilon
$$

for all $x \in G_{1}$ ? When this problem has a solution, we say that the homomorphisms from $G_{1}$ to $G_{2}$ are stable. In the next year, D. H. Hyers [6] gave a partial solution of Ulam's problem for the case of approximate additive mappings under the assumption that $G_{1}$ and $G_{2}$ are Banach spaces. Hyers' result was generalized by T. Aoki [1] for additive mappings and by Th. M. Rassias [17] for linear mappings by considering the stability problem with unbounded Cauchy differences. The paper of Th. M. Rassias had much influence in the development of stability problems. The terminology Hyers-Ulam-Rassias stability originated from this historical background. During the last decades, the stability problems of

Received October 26, 2011. Accepted November 22, 2011.

2000 Mathematics Subject Classification. Primary 39B52.

Key words and phrases. Hyers-Ulam-Rassias stability, fixed point method, mixed type functional equation . 
functional equations have been extensively investigated by a number of mathematicians, see [5], [7], [9]-[14].

Almost all subsequent proofs, in this very active area, have used Hyers' method of [6]. Namely, the mapping $F$, which is the solution of a functional equation, is explicitly constructed, starting from the given mapping $f$, by the formulae $F(x)=\lim _{n \rightarrow \infty} \frac{1}{2^{n}} f\left(2^{n} x\right)$ or $F(x)=\lim _{n \rightarrow \infty} 2^{n} f\left(\frac{x}{2^{n}}\right)$. We call it a direct method. In 2003, L. Cădariu and V. Radu [2] observed that the existence of the solution $F$ for a functional equation and the estimation of the difference with the given mapping $f$ can be obtained from the fixed point theory alternative. This method is called a fixed point method. In 2004, they [4] applied this method to prove stability theorems of the Cauchy functional equation:

$$
f(x+y)-f(x)-f(y)=0 .
$$

In 2003, they [3] obtained the stability of the quadratic functional equation:

$$
f(x+y)+f(x-y)-2 f(x)-2 f(y)=0
$$

by using the fixed point method. Notice that if we consider the mappings $f_{1}, f_{2}: \mathbb{R} \rightarrow \mathbb{R}$ defined by $f_{1}(x)=a x$ and $f_{2}(x)=a x^{2}$, where $a$ is a real constant, then $f_{1}$ satisfies the equation (1.1) and $f_{2}$ holds (1.2), respectively. We say a solution of (1.1) an additive mapping and a mapping satisfying (1.2) is called a quadratic mapping. Now we consider the following functional equation:

$$
f(x+y+z)-f(x+y)-f(y+z)-f(x+z)+f(x)+f(y)+f(z)=0
$$

which is called the mixed type functional equation. The mapping $f: \mathbb{R} \rightarrow$ $\mathbb{R}$ defined by $f(x)=a x^{2}+b x$ satisfies this functional equation, where $a, b$ are real constants. We call a solution of (1.3) a quadratic-additive mapping. In 2002, S.-M. Jung [8] obtained a stability of the functional equation (1.3) by handling the odd part and the even part of the given mapping $f$, respectively (see also [16]). In his processing, he needed to take an additive mapping $A$ which is close to the odd part $\frac{f(x)-f(-x)}{2}$ of $f$ and a quadratic mapping $Q$ which is approximate to the even part $\frac{f(x)+f(-x)}{2}$ of it, and then combining $A$ and $Q$ to prove the existence of a quadratic-additive mapping $F$ which is close to the given mapping $f$.

In this paper, we will prove the stability of the quadratic-additive functional equation (1.3) by using the fixed point theory. In the previous results of stability problems of (1.3), as we mentioned above, he had to get a solution by using the direct method to the odd part and even part, respectively. Instead of splitting the given mapping $f: X \rightarrow Y$ into 
two parts, in this paper, we can take the desired solution $F$ at once. Precisely, we introduce a strictly contractive mapping with Liptshitz constant $0<L<1$. Using the fixed point theory in the sense of $L$. Cădariu and V. Radu, together with suitable conditions, we can show that the contractive mapping has the fixed point. Actually the fixed point $F$ becomes the precise solution of (1.3). In section 2, we prove several stability results of the functional equation (1.3) using the fixed point theory, see Theorem 2.3, Theorem 2.4, and Theorem 2.5. In section 3 , we use the results in the previous sections to get a stability of the Cauchy functional equation (1.1) and that of the quadratic functional equation (1.2), respectively.

\section{Main results}

We recall the following result of the fixed point theory by Margolis and Diaz.

Theorem 2.1. ( [15] or [18]) Suppose that a complete generalized metric space $(X, d)$, which means that the metric $d$ may assume infinite values, and a strictly contractive mapping $J: X \rightarrow X$ with the Lipschitz constant $0<L<1$ are given. Then, for each given element $x \in X$, either

$$
d\left(J^{n} x, J^{n+1} x\right)=+\infty, \forall n \in \mathbb{N} \cup\{0\},
$$

or there exists a nonnegative integer $k$ such that:

(1) $d\left(J^{n} x, J^{n+1} x\right)<+\infty$ for all $n \geq k$;

(2) the sequence $\left\{J^{n} x\right\}$ is convergent to a fixed point $y^{*}$ of $J$;

(3) $y^{*}$ is the unique fixed point of $J$ in $Y:=\left\{y \in X, d\left(J^{k} x, y\right)<+\infty\right\}$;

(4) $d\left(y, y^{*}\right) \leq(1 /(1-L)) d(y, J y)$ for all $y \in Y$.

Throughout this paper, let $\mathrm{V}$ be a (real or complex) linear space and $Y$ a Banach space. For a given mapping $f: V \rightarrow Y$, we use the following abbreviation

$D f(x, y, z):=f(x+y+z)-f(x+y)-f(y+z)-f(x+z)+f(x)+f(y)+f(z)$

for all $x, y, z \in V$. If $f$ is a solution of the functional equation $D f \equiv 0$, see (1.3), we call it a quadratic-additive mapping. We first prove the following lemma. 
Lemma 2.2. If $f: V \rightarrow Y$ is a mapping such that $D f(x, y, z)=0$ for all $x, y, z \in V \backslash\{0\}$, then

$$
D f(x, y, z)=0
$$

for all $x, y, z \in V$.

Proof. By choosing $x \in V \backslash\{0\}$, we get

$$
f(0)=\frac{D f(2 x,-x,-x)+D f(-2 x, x, x)}{2}=0 .
$$

So we can compute that $D f(x, y, 0)=0, D f(x, 0, z)=0, D f(0, y, z)=$ $0, D f(x, 0,0)=0, D f(0,0, z)=0, D f(0, y, 0)=0$ and $D f(0,0,0)=0$ for all $x, y, z \in V \backslash\{0\}$ as we desired.

In the following theorem, we can prove the stability of the functional equation (1.3) using the fixed point theory.

Theorem 2.3. Let $f: V \rightarrow Y$ be a mapping for which there exists a mapping $\varphi:(V \backslash\{0\})^{3} \rightarrow[0, \infty)$ such that

$$
\|D f(x, y, z)\| \leq \varphi(x, y, z)
$$

for all $x, y, z \in V \backslash\{0\}$. If there exist constants $0<L, L^{\prime}<1$ such that $\varphi$ has the property

$$
\frac{1}{L^{\prime}} \varphi(x, y, z)<\varphi(2 x, 2 y, 2 z) \leq 2 L \varphi(x, y, z)
$$

for all $x, y, z \in V \backslash\{0\}$, then there exists a unique quadratic-additive mapping $F: V \rightarrow Y$ such that

$$
\|f(x)-F(x)\| \leq \frac{3(\varphi(x, x,-x)+\varphi(-x,-x, x))}{8(1-L)}
$$

for all $x \in V \backslash\{0\}$. In particular, $F$ is represented by

$$
F(x)=\lim _{n \rightarrow \infty}\left(\frac{f\left(2^{n} x\right)+f\left(-2^{n} x\right)}{2 \cdot 4^{n}}+\frac{f\left(2^{n} x\right)-f\left(-2^{n} x\right)}{2^{n+1}}\right)
$$

for all $x \in V$.

Proof. It follows from (2.2) that

$$
\lim _{n \rightarrow \infty} \frac{\varphi\left(2^{n} x, 2^{n} y, 2^{n} z\right)}{2^{n}}=\lim _{n \rightarrow \infty} L^{n} \varphi(x, y, z)=0
$$


for all $x, y, z \in V \backslash\{0\}$ and

$$
\begin{aligned}
\|2 f(0)\| & =\left\|D f\left(\frac{2 x}{2^{n}},-\frac{x}{2^{n}},-\frac{x}{2^{n}}\right)+D f\left(-\frac{2 x}{2^{n}}, \frac{x}{2^{n}}, \frac{x}{2^{n}}\right)\right\| \\
& \leq\left(\varphi\left(\frac{2 x}{2^{n}},-\frac{x}{2^{n}},-\frac{x}{2^{n}}\right)+\varphi\left(-\frac{2 x}{2^{n}}, \frac{x}{2^{n}}, \frac{x}{2^{n}}\right)\right) \\
& \leq L^{\prime n}(\varphi(2 x,-x,-x)+\varphi(-2 x, x, x))
\end{aligned}
$$

for all $n \in \mathbb{N}$ and for any fixed $x \in V \backslash\{0\}$. From this, we know that $f(0)=0$ as $n \rightarrow \infty$. Let $S$ be the set of all mappings $g: V \rightarrow Y$ with $g(0)=0$. We introduce a generalized metric on $S$ by

$$
\begin{array}{r}
d(g, h)=\inf \left\{K \in \mathbb{R}^{+} \mid\|g(x)-h(x)\| \leq K(\varphi(x, x,-x)+\varphi(-x,-x, x))\right. \\
\text { for all } x \in V \backslash\{0\}\} .
\end{array}
$$

It is easy to show that $(S, d)$ is a generalized complete metric space. Now we consider the mapping $J: S \rightarrow S$, which is defined by

$$
J g(x):=\frac{g(2 x)-g(-2 x)}{4}+\frac{g(2 x)+g(-2 x)}{8}
$$

for all $x \in V$. Notice

$$
J^{n} g(x)=\frac{g\left(2^{n} x\right)-g\left(-2^{n} x\right)}{2^{n+1}}+\frac{g\left(2^{n} x\right)+g\left(-2^{n} x\right)}{2 \cdot 4^{n}}
$$

for all $n \in \mathbb{N}$ and $x \in V$. Let $g, h \in S$ and let $K \in[0, \infty]$ be an arbitrary constant with $d(g, h) \leq K$. From the definition of $d$, we have

$$
\begin{aligned}
\|J g(x)-J h(x)\| & \leq \frac{3}{8}\|g(2 x)-h(2 x)\|+\frac{1}{8}\|g(-2 x)-h(-2 x)\| \\
& \leq \frac{K}{2}(\varphi(2 x, 2 x,-2 x)+\varphi(-2 x,-2 x, 2 x)) \\
& \leq L K(\varphi(x, x,-x)+\varphi(-x,-x, x))
\end{aligned}
$$

for all $x \in V \backslash\{0\}$, which implies that

$$
d(J g, J h) \leq L d(g, h)
$$

for any $g, h \in S$. That is, $J$ is a strictly contractive self-mapping of $S$ with the Lipschitz constant $0<L<1$. Moreover, by (2.1) we see that

$$
\begin{aligned}
\|f(x)-J f(x)\| & =\left\|\frac{3 D f(x, x,-x)-D f(-x,-x, x)}{8}\right\| \\
& \leq \frac{3(\varphi(x, x,-x)+\varphi(-x,-x, x))}{8}
\end{aligned}
$$

for all $x \in V \backslash\{0\}$. It means that $d(f, J f) \leq \frac{3}{8}<\infty$ by the definition of $d$. Therefore according to Theorem 2.1, the sequence $\left\{J^{n} f\right\}$ converges 
to the unique fixed point $F: V \rightarrow Y$ of $J$ in the set $T=\{g \in S \mid d(f, g)<$ $\infty$ \}, which is represented by (2.4) for all $x \in V$. Notice that

$$
d(f, F) \leq \frac{1}{1-L} d(f, J f) \leq \frac{3}{8(1-L)}
$$

which implies (2.3). By the definition of $F$, together with (2.1) and (2.4), we have

$$
\begin{aligned}
\|D F(x, y, z)\|= & \lim _{n \rightarrow \infty} \| \frac{D f\left(2^{n} x, 2^{n} y, 2^{n} z\right)-D f\left(-2^{n} x,-, 2^{n} y,-2^{n} z\right)}{2^{n+1}} \\
& +\frac{D f\left(2^{n} x, 2^{n} y, 2^{n} z\right)+D f\left(-2^{n} x,-2^{n} y,-2^{n} z\right)}{2 \cdot 4^{n}} \| \\
\leq & \lim _{n \rightarrow \infty} \frac{2^{n}+1}{2 \cdot 4^{n}}\left(\varphi\left(2^{n} x, 2^{n} y, 2^{n} z\right)+\varphi\left(-2^{n} x,-2^{n} y,-2^{n} z\right)\right) \\
=0 &
\end{aligned}
$$

for all $x, y, z \in V \backslash\{0\}$. By Lemma 2.2, we have proved that

$$
D F(x, y, z)=0
$$

for all $x, y, z \in V$.

Theorem 2.4. Let $\varphi:(V \backslash\{0\})^{3} \rightarrow[0, \infty)$ be a mapping satisfying

$$
\varphi(2 x, 2 y, 2 z) \leq 2 L \varphi(x, y, z)
$$

for all $x, y, z \in V \backslash\{0\}$ with $0<L<\frac{1}{2}$. If $f: V \rightarrow Y$ satisfies the inequality (2.1) for all $x, y, z \in V \backslash\{0\}$, then there exists a unique quadraticadditive mapping $F: V \rightarrow Y$ satisfying (2.3) for all $x \in V \backslash\{0\}$. Moreover, if $\varphi$ is continuous, then $f$ is itself a quadratic-additive mapping.

Proof. From (2.5), we have

$$
\begin{aligned}
\|2 f(0)\| & =\lim _{n \rightarrow \infty}\left\|D f\left(2^{n+1} x,-2^{n} x,-2^{n} x\right)+D f\left(-2^{n+1} x, 2^{n} x, 2^{n} x\right)\right\| \\
& \leq \lim _{n \rightarrow \infty}\left(\varphi\left(2^{n+1} x,-2^{n} x,-2^{n} x\right)+D f\left(-2^{n+1} x, 2^{n} x, 2^{n} x\right)\right) \\
& \leq \lim _{n \rightarrow \infty}(2 L)^{n}(\varphi(2 x,-x,-x)+\varphi(-2 x, x, x))=0
\end{aligned}
$$

for all $x \in V \backslash\{0\}$. By the same method used in the proof of Theorem 2.3 , we know that there exists a unique quadratic-additive mapping $F$ : $V \rightarrow Y$ satisfying (2.3) for all $x \in V \backslash\{0\}$. Since $\varphi$ is continuous, we get

$$
\begin{aligned}
\lim _{n \rightarrow \infty} \varphi\left(\left(a_{1} \cdot 2^{n}+a_{2}\right) x,\left(b_{1} \cdot 2^{n}+b_{2}\right) y,\left(c_{1} \cdot 2^{n}+c_{2}\right) z\right) \\
\leq \lim _{n \rightarrow \infty}(2 L)^{n} \varphi\left(\left(a_{1}+\frac{a_{2}}{2^{n}}\right) x,\left(b_{1}+\frac{b_{2}}{2^{n}}\right) y,\left(c_{1}+\frac{c_{2}}{2^{n}}\right) z\right) \\
\quad=0 \cdot \varphi\left(a_{1} x, b_{1} y, c_{1} z\right)=0
\end{aligned}
$$


for all $x, y, z \in V \backslash\{0\}$ and for any fixed integers $a_{1}, a_{2}, b_{1}, b_{2}, c_{1}, c_{2}$ with $a_{1}, b_{1}, c_{1} \neq 0$. Therefore, we obtain

$$
\begin{aligned}
\|F(x)-f(x)\| \leq & \lim _{n \rightarrow \infty}\left(\| D f\left(\left(2^{n}+1\right) x, 2^{n} x,-2^{n} x\right)-D F\left(\left(2^{n}+1\right) x, 2^{n} x,\right.\right. \\
& \left.-2^{n} x\right)\|+2\|(F-f)\left(\left(2^{n}+1\right) x\right)\|+\|(f-F)\left(\left(2^{n+1}+1\right) x\right) \| \\
& \left.+\left\|(F-f)\left(2^{n} x\right)\right\|+\left\|(F-f)\left(-2^{n} x\right)\right\|\right) \\
\leq & \lim _{n \rightarrow \infty}\left(\varphi\left(\left(2^{n}+1\right) x, 2^{n} x,-2^{n} x\right)\right. \\
& \left.+\frac{3}{8(1-L)}\left(2 \psi\left(\left(2^{n}+1\right) x\right)+2 \psi\left(2^{n} x\right)+\psi\left(\left(2^{n+1}+1\right) x\right)\right)\right) \\
= & 0
\end{aligned}
$$

for all $x \in V \backslash\{0\}$, where $\psi(x)$ is defined by $\psi(x)=\varphi(x, x,-x)+$ $\varphi(-x,-x, x)$. Since $f(0)=0=F(0)$, we have shown that $f \equiv F$. This completes the proof of this theorem.

We continue our investigation with the next result.

Theorem 2.5. Let $\varphi:(V \backslash\{0\})^{3} \rightarrow[0, \infty)$. Suppose that $f: V \rightarrow Y$ satisfies the inequality $\|D f(x, y, z)\| \leq \varphi(x, y, z)$ for all $x, y, z \in V \backslash\{0\}$. If there exists $0<L<1$ such that the mapping $\varphi$ has the property

$$
L \varphi(2 x, 2 y, 2 z) \geq 4 \varphi(x, y, z)
$$

for all $x, y, z \in V \backslash\{0\}$, then there exists a unique quadratic-additive mapping $F: V \rightarrow Y$ such that

$$
\|f(x)-F(x)\| \leq \frac{L}{4(1-L)}(\varphi(x, x,-x)+\varphi(-x,-x, x))
$$

for all $x \in V \backslash\{0\}$. In particular, $F$ is represented by

$F(x)=\lim _{n \rightarrow \infty}\left(2^{n-1}\left(f\left(\frac{x}{2^{n}}\right)-f\left(-\frac{x}{2^{n}}\right)\right)+\frac{4^{n}}{2}\left(f\left(\frac{x}{2^{n}}\right)+f\left(-\frac{x}{2^{n}}\right)\right)\right)$

for all $x \in V$.

Proof. It follows from (2.6) that

$$
\begin{aligned}
\|2 f(0)\| & =\lim _{n \rightarrow \infty}\left\|D f\left(\frac{2 x}{2^{n}},-\frac{x}{2^{n}},-\frac{x}{2^{n}}\right)+D f\left(-\frac{2 x}{2^{n}}, \frac{x}{2^{n}}, \frac{x}{2^{n}}\right)\right\| \\
& \leq \lim _{n \rightarrow \infty}\left(\varphi\left(\frac{2 x}{2^{n}},-\frac{x}{2^{n}},-\frac{x}{2^{n}}\right)+\varphi\left(-\frac{2 x}{2^{n}}, \frac{x}{2^{n}}, \frac{x}{2^{n}}\right)\right) \\
& \leq \lim _{n \rightarrow \infty}\left(\frac{L}{4}\right)^{n}(\varphi(2 x,-x,-x)+\varphi(-2 x, x, x))=0
\end{aligned}
$$


for an fixed $x \in V \backslash\{0\}$. From this, we know that $f(0)=0$. Let the set $(S, d)$ be as in the proof of Theorem 2.3. Now we consider the mapping $J: S \rightarrow S$ defined by

$$
J g(x):=g\left(\frac{x}{2}\right)-g\left(-\frac{x}{2}\right)+2\left(g\left(\frac{x}{2}\right)+g\left(-\frac{x}{2}\right)\right)
$$

for all $g \in S$ and $x \in V$. Notice that

$$
J^{n} g(x)=2^{n-1}\left(g\left(\frac{x}{2^{n}}\right)-g\left(-\frac{x}{2^{n}}\right)\right)+\frac{4^{n}}{2}\left(g\left(\frac{x}{2^{n}}\right)+g\left(-\frac{x}{2^{n}}\right)\right)
$$

and $J^{0} g(x)=g(x)$ for all $x \in V$. Let $g, h \in S$ and let $K \in[0, \infty]$ be an arbitrary constant with $d(g, h) \leq K$. From the definition of $d$, we have

$$
\begin{aligned}
\|J g(x)-J h(x)\| & =3\left\|g\left(\frac{x}{2}\right)-h\left(\frac{x}{2}\right)\right\|+\left\|g\left(-\frac{x}{2}\right)-h\left(-\frac{x}{2}\right)\right\| \\
& \leq 4 K\left(\varphi\left(\frac{x}{2}, \frac{x}{2},-\frac{x}{2}\right)+\varphi\left(-\frac{x}{2},-\frac{x}{2}, \frac{x}{2}\right)\right) \\
& \leq L K(\varphi(x, x,-x)+\varphi(-x,-x, x))
\end{aligned}
$$

for all $x \in V \backslash\{0\}$. So

$$
d(J g, J h) \leq L d(g, h)
$$

for any $g, h \in S$. That is, $J$ is a strictly contractive self-mapping of $S$ with the Lipschitz constant $0<L<1$. Also we see that

$$
\begin{aligned}
\|f(x)-J f(x)\| & =\left\|-D f\left(\frac{x}{2}, \frac{x}{2},-\frac{x}{2}\right)\right\| \\
& \leq \varphi\left(\frac{x}{2}, \frac{x}{2},-\frac{x}{2}\right) \\
& \leq \frac{L}{4}(\varphi(x, x,-x)+\varphi(-x,-x, x))
\end{aligned}
$$

for all $x \in V \backslash\{0\}$, which implies that $d(f, J f) \leq \frac{L}{4}<\infty$. Therefore according to Theorem 2.1, the sequence $\left\{J^{n} f\right\}$ converges to the unique fixed point $F$ of $J$ in the set $T:=\{g \in S \mid d(f, g)<\infty\}$, which is represented by (2.8). Since

$$
d(f, F) \leq \frac{1}{1-L} d(f, J f) \leq \frac{L}{4(1-L)}
$$


the inequality (2.7) holds. From the definition of $F(x),(2.1)$ and (2.6), we have

$$
\begin{aligned}
\|D F(x, y, z)\|= & \lim _{n \rightarrow \infty} \| 2^{n-1}\left(D f\left(\frac{x}{2^{n}}, \frac{y}{2^{n}}, \frac{z}{2^{n}}\right)-D f\left(-\frac{x}{2^{n}},-\frac{y}{2^{n}},-\frac{z}{2^{n}}\right)\right) \\
& +\frac{4^{n}}{2}\left(D f\left(\frac{x}{2^{n}}, \frac{y}{2^{n}}, \frac{z}{2^{n}}\right)+D f\left(-\frac{x}{2^{n}},-\frac{y}{2^{n}},-\frac{z}{2^{n}}\right)\right) \| \\
\leq & \lim _{n \rightarrow \infty} \frac{2^{n}+4^{n}}{2}\left(\varphi\left(\frac{x}{2^{n}}, \frac{y}{2^{n}}, \frac{z}{2^{n}}\right)+\varphi\left(-\frac{x}{2^{n}},-\frac{y}{2^{n}},-\frac{z}{2^{n}}\right)\right) \\
= & 0
\end{aligned}
$$

for all $x, y, z \in V \backslash\{0\}$. By Lemma 2.2, $F$ is quadratic-additive.

Remark 2.6. If $\varphi$ satisfies the additional condition $\varphi(x, y, z)=$ $\varphi(-x,-y,-z)$ for all $x, y, z \in V \backslash\{0\}$ in Theorem 2.3, Theorem 2.4, and Theorem 2.5, then the inequalities (2.3) and (2.7) can be replace by

$$
\|f(x)-F(x)\| \leq \frac{\varphi(x, x,-x)}{2(1-L)}
$$

and

$$
\|f(x)-F(x)\| \leq \frac{L}{4(1-L)} \varphi(x, x,-x)
$$

for all $x \in V \backslash\{0\}$, respectively.

\section{Applications}

For a given mapping $f: V \rightarrow Y$, we use the following abbreviations

$$
\begin{aligned}
& A f(x, y):=f(x+y)-f(x)-f(y), \\
& Q f(x, y):=f(x+y)+f(x-y)-2 f(x)-2 f(y)
\end{aligned}
$$

for all $x, y \in V$. Using Theorem 2.3, Theorem 2.4, and Theorem 2.5, we will show the stability results of the additive functional equation $A f \equiv 0$ and the quadratic functional equation $Q f \equiv 0$ in the following corollaries.

Corollary 3.1. Let $f_{i}: V \rightarrow Y, i=1,2,3$, be mappings for which there exist mappings $\phi_{i}: V^{2} \rightarrow[0, \infty), i=1,2,3$, such that

$$
\left\|A f_{i}(x, y)\right\| \leq \phi_{i}(x, y)
$$

for all $x, y \in V$, respectively. If there exist $0<L, L^{\prime}<1$ such that

$$
\begin{gathered}
\frac{1}{L^{\prime}} \phi_{1}(x, y) \leq \phi_{1}(2 x, 2 y) \leq 2 L \phi_{1}(x, y), \\
\phi_{2}(2 x, 2 y) \leq L \phi_{2}(x, y),
\end{gathered}
$$




$$
4 \phi_{3}(x, y) \leq L \phi_{3}(2 x, 2 y)
$$

for all $x, y \in V$, then there exist unique additive mappings $F_{i}: V \rightarrow$ $Y, i=1,2,3$, such that

$$
\begin{aligned}
& \left\|f_{1}(x)-F_{1}(x)\right\| \leq \frac{3 \Phi_{1}(x)}{8(1-L)}, \\
& \left\|f_{2}(x)-F_{2}(x)\right\| \leq \frac{3 \Phi_{2}(x)}{8\left(1-\frac{L}{2}\right)}, \\
& \left\|f_{3}(x)-F_{3}(x)\right\| \leq \frac{L \Phi_{3}(x)}{4(1-L)}
\end{aligned}
$$

for all $x \in V$, where $\Phi_{i}: V \rightarrow Y, i=1,2,3$, are defined by

$$
\Phi_{i}(x)=\phi_{i}(2 x,-x)+2 \phi_{i}(x,-x)+\phi_{i}(-2 x, x)+2 \phi_{i}(-x, x)
$$

for all $x \in V$. In particular, the mappings $F_{1}, F_{2}, F_{3}$ are represented by

$$
\begin{gathered}
F_{1}(x)=\lim _{n \rightarrow \infty} \frac{f_{1}\left(2^{n} x\right)}{2^{n}}, \\
F_{2}(x)=\lim _{n \rightarrow \infty} \frac{f_{2}\left(2^{n} x\right)}{2^{n}}, \\
F_{3}(x)=\lim _{n \rightarrow \infty} 2^{n} f_{3}\left(\frac{x}{2^{n}}\right)
\end{gathered}
$$

for all $x \in V$. Moreover, if $\phi_{2}(x, y)$ is continuous then $f_{2}$ is itself an additive mapping.

Proof. Notice that

$$
D f_{i}(x, y, z)=A f_{i}(x+y, z)-A f_{i}(x, z)-A f_{i}(y, z)
$$

for all $x, y, z \in V$ and $i=1,2,3$. Put

$$
\varphi_{i}(x, y, z):=\phi_{i}(x+y, z)+\phi_{i}(x, z)+\phi_{i}(y, z)
$$

for all $x, y, z \in V$ and $i=1,2,3$, then $\varphi_{1}$ satisfies (2.2), $\varphi_{2}$ satisfies (2.5), and $\varphi_{3}$ satisfies (2.6). Therefore, according to Theorem 2.3, there exists a unique mapping $F_{1}: V \rightarrow Y$ satisfying (3.5), which is represented by 
(2.4). Observe that, by (3.1) and (3.2),

$$
\begin{aligned}
\lim _{n \rightarrow \infty}\left\|\frac{f_{1}\left(2^{n} x\right)+f_{1}\left(-2^{n} x\right)}{2^{n+1}}\right\| & =\lim _{n \rightarrow \infty}\left\|\frac{f_{1}\left(2^{n} x\right)+f_{1}\left(-2^{n} x\right)-f_{1}(0)}{2^{n+1}}\right\| \\
& =\lim _{n \rightarrow \infty} \frac{1}{2^{n+1}}\left\|A f_{1}\left(2^{n} x,-2^{n} x\right)\right\| \\
& \leq \lim _{n \rightarrow \infty} \frac{1}{2^{n+1}} \phi_{1}\left(2^{n} x,-2^{n} x\right) \\
& \leq \lim _{n \rightarrow \infty} \frac{L^{n}}{2} \phi_{1}(x,-x)=0
\end{aligned}
$$

as well as

$$
\lim _{n \rightarrow \infty}\left\|\frac{f_{1}\left(2^{n} x\right)+f_{1}\left(-2^{n} x\right)}{2 \cdot 4^{n}}\right\| \leq \lim _{n \rightarrow \infty} \frac{2^{n} L^{n}}{2 \cdot 4^{n}} \phi_{1}(x,-x)=0
$$

for all $x \in V$. From this and (2.4), we get (3.7). Moreover, we have

$$
\left\|\frac{A f_{1}\left(2^{n} x, 2^{n} y\right)}{2^{n}}\right\| \leq \frac{\phi_{1}\left(2^{n} x, 2^{n} y\right)}{2^{n}} \leq L^{n} \phi_{1}(x, y)
$$

for all $x, y \in V$. Taking the limit as $n \rightarrow \infty$ in the above inequality, we get

$$
A F_{1}(x, y)=0
$$

for all $x, y \in V$. According to Theorem 2.4, there exists a unique mapping $F_{2}: V \rightarrow Y$ satisfying (3.6), which is represented by (2.4). By using the similar method to prove (3.8), we can show that $F_{2}$ is represented by (3.9). In particular, if $\phi_{2}(x, y)$ is continuous, then $\varphi_{2}$ is continuous on $(V \backslash\{0\})^{3}$ and we can say that $f_{2}$ is a quadratic additive mapping by Theorem 2.4. On the other hand, according to Theorem 2.5, there exists a unique mapping $F_{3}: V \rightarrow Y$ satisfying (3.7), which is represented by (2.8). Observe that

$$
\begin{aligned}
\lim _{n \rightarrow \infty} 2^{2 n-1}\left\|f_{3}\left(\frac{x}{2^{n}}\right)+f_{3}\left(\frac{-x}{2^{n}}\right)\right\| & =\lim _{n \rightarrow \infty} 2^{2 n-1}\left\|A f_{3}\left(\frac{x}{2^{n}},-\frac{x}{2^{n}}\right)\right\| \\
& \leq \lim _{n \rightarrow \infty} 2^{2 n-1} \phi_{3}\left(\frac{x}{2^{n}},-\frac{x}{2^{n}}\right) \\
& \leq \lim _{n \rightarrow \infty} \frac{L^{n}}{2} \phi_{3}(x,-x)=0
\end{aligned}
$$

as well as

$$
\lim _{n \rightarrow \infty} 2^{n-1}\left\|f_{3}\left(\frac{x}{2^{n}}\right)+f_{3}\left(\frac{-x}{2^{n}}\right)\right\| \leq \lim _{n \rightarrow \infty} \frac{L^{n}}{2^{n+1}} \phi_{3}(x,-x)=0
$$


for all $x \in V$. From these and (2.8), we get (3.10). Moreover, we have

$$
\left\|2^{n} A f_{3}\left(\frac{x}{2^{n}}, \frac{y}{2^{n}}\right)\right\| \leq 2^{n} \phi_{3}\left(\frac{x}{2^{n}}, \frac{y}{2^{n}}\right) \leq \frac{L^{n}}{2^{n}} \phi_{3}(x, y)
$$

for all $x, y \in V$. Taking the limit as $n \rightarrow \infty$ in the above inequality, we get

$$
A F_{3}(x, y)=0
$$

for all $x, y \in V$.

Corollary 3.2. Let $\phi_{i}: V^{2} \rightarrow[0, \infty), i=1,2,3$, be given mappings. Suppose that each $f_{i}: V \rightarrow Y, i=1,2,3$, satisfies

$$
\left\|Q f_{i}(x, y)\right\| \leq \phi_{i}(x, y)
$$

for all $x, y \in V$, respectively. If there exists $L<1$ such that the mapping $\phi_{1}$ satisfies (3.2), $\phi_{2}$ satisfies (3.3) and $\phi_{3}$ satisfies (3.4) for all $x, y \in V$, then we have unique quadratic mappings $F_{1}, F_{2}, F_{3}: V \rightarrow Y$ such that

$$
\begin{gathered}
\left\|f_{1}(x)-F_{1}(x)\right\| \leq \frac{3 \Phi_{1}(x)}{16(1-L)}, \\
\left\|f_{2}(x)-F_{2}(x)\right\| \leq \frac{3 \Phi_{2}(x)}{16\left(1-\frac{L}{2}\right)}, \\
\left\|f_{3}(x)-F_{3}(x)\right\| \leq \frac{L \Phi_{3}(x)}{8(1-L)}
\end{gathered}
$$

for all $x \in V$, where $\Phi_{i}: V \rightarrow Y, i=1,2,3$, are defined by

$$
\begin{aligned}
\Phi_{i}(x)= & \phi_{i}(2 x,-x)+\phi_{i}(x, 0)+\phi_{i}(0, x)+2 \phi_{i}(x,-x) \\
& +\phi_{i}(-2 x, x)+\phi_{i}(-x, 0)+\phi_{i}(0,-x)+2 \phi_{i}(-x, x) .
\end{aligned}
$$

In particular, $F_{1}, F_{2}, F_{3}$ are represented by

$$
\begin{aligned}
& F_{1}(x)=\lim _{n \rightarrow \infty} \frac{f_{1}\left(2^{n} x\right)}{4^{n}}, \\
& F_{2}(x)=\lim _{n \rightarrow \infty} \frac{f_{2}\left(2^{n} x\right)}{4^{n}}, \\
& F_{3}(x)=\lim _{n \rightarrow \infty} 4^{n} f_{3}\left(\frac{x}{2^{n}}\right)
\end{aligned}
$$

for all $x \in V$. Moreover, if $\phi_{2}(x, y)$ is continuous, then $f_{2}$ is itself a quadratic mapping. 
Proof. Notice that

$$
D f_{i}(x, y, z)=\frac{1}{2}\left(Q f_{i}(x+y, z)-Q f_{i}(x-z, y)+Q f_{i}(x, y+z)\right)-Q f_{i}(x, z)
$$

for all $x, y, z \in V$ and $i=1,2,3$. Put

$$
\varphi_{i}(x, y, z):=\frac{1}{2}\left(\phi_{i}(x+y, z)+\phi_{i}(x, y+z)+\phi_{i}(x-z, y)\right)+\phi_{i}(x, z)
$$

for all $x, y, z \in V$ and $i=1,2,3$, then $\varphi_{1}$ satisfies (2.2), $\varphi_{2}$ satisfies (2.5), and $\varphi_{3}$ satisfies (2.6). Moreover, we have

$$
\left\|D f_{i}(x, y, z)\right\| \leq \varphi_{i}(x, y, z)
$$

for all $x, y, z \in V$ and $i=1,2,3$. According to Theorem 2.3, there exists a unique mapping $F_{1}: V \rightarrow Y$ satisfying (3.11) which is represented by (2.4). Observe that

$$
\begin{aligned}
\lim _{n \rightarrow \infty}\left\|\frac{f_{1}\left(2^{n} x\right)-f_{1}\left(-2^{n} x\right)}{2^{n+1}}\right\| & =\lim _{n \rightarrow \infty} \frac{1}{2^{n+1}}\left\|Q f_{1}\left(0,2^{n} x\right)\right\| \\
& \leq \lim _{n \rightarrow \infty} \frac{1}{2^{n+1}} \phi_{1}\left(0,2^{n} x\right) \\
& \leq \lim _{n \rightarrow \infty} \frac{L^{n}}{2} \phi_{1}(0, x)=0
\end{aligned}
$$

as well as

$$
\lim _{n \rightarrow \infty}\left\|\frac{f_{1}\left(2^{n} x\right)-f_{1}\left(-2^{n} x\right)}{2 \cdot 4^{n}}\right\| \leq \lim _{n \rightarrow \infty} \frac{L^{n}}{2^{n+1}} \phi_{1}(0, x)=0
$$

for all $x \in V$. From this and (2.4), we get (3.14) for all $x \in V$. Moreover, we have

$$
\left\|\frac{Q f_{1}\left(2^{n} x, 2^{n} y\right)}{4^{n}}\right\| \leq \frac{\phi_{1}\left(2^{n} x, 2^{n} y\right)}{4^{n}} \leq \frac{L^{n}}{2^{n}} \phi_{1}(x, y)
$$

for all $x, y \in V$. Taking the limit as $n \rightarrow \infty$ in the above inequality, we get

$$
Q F_{1}(x, y)=0
$$

for all $x, y \in V$. According to Theorem 2.4, there exists a unique mapping $F_{2}: V \rightarrow Y$ satisfying (3.12), which is represented by (2.4). By using the similar method to prove (3.14), we can show that $F_{2}$ is represented by (3.15). In particular, $\phi_{2}(x, y)$ is continuous, then $\varphi_{2}$ is continuous on $(V \backslash\{0\})^{3}$ and we can say that $f_{2}$ is a quadratic additive mapping by Theorem 2.4. On the other hand, according to Theorem 2.5 , there exists a unique mapping $F_{3}: V \rightarrow Y$ satisfying (3.13) which is represented by (2.8). Observe that

$$
4^{n}\left\|f_{3}\left(\frac{x}{2^{n}}\right)-f_{3}\left(-\frac{x}{2^{n}}\right)\right\|=4^{n}\left\|Q f_{3}\left(0, \frac{x}{2^{n}}\right)\right\| \leq 4^{n} \phi_{3}\left(0, \frac{x}{2^{n}}\right) \leq L^{n} \phi_{3}(0, x)
$$


for all $x \in V$. It leads us to get

$\lim _{n \rightarrow \infty} 4^{n}\left(f_{3}\left(\frac{x}{2^{n}}\right)-f_{3}\left(-\frac{x}{2^{n}}\right)\right)=0$ and $\lim _{n \rightarrow \infty} 2^{n}\left(f_{3}\left(\frac{x}{2^{n}}\right)-f_{3}\left(-\frac{x}{2^{n}}\right)\right)=0$ for all $x \in V$. From these and (2.8), we obtain (3.16). Moreover, we have

$$
\left\|4^{n} Q f_{3}\left(\frac{x}{2^{n}}, \frac{y}{2^{n}}\right)\right\| \leq 4^{n} \phi_{3}\left(\frac{x}{2^{n}}, \frac{y}{2^{n}}\right) \leq L^{n} \phi_{3}(x, y)
$$

for all $x, y \in V$. Taking the limit as $n \rightarrow \infty$ in the above inequality, we get

for all $x, y \in V$.

$$
Q F_{3}(x, y)=0
$$

Now, we obtain Hyers-Ulam-Rassias stability results in the framework of normed spaces using Theorem 2.3, Theorem 2.4, and Theorem 2.5.

Corollary 3.3. Let $X$ be a normed space. Suppose that the mapping $f: X \rightarrow Y$ satisfies the inequality

$$
\|D f(x, y, z)\| \leq \theta\left(\|x\|^{p}+\|y\|^{p}+\|z\|^{p}\right)
$$

for all $x, y, z \in X \backslash\{0\}$, where $\theta \geq 0$ and $p \in(-\infty, 0) \cup(0,1) \cup(2, \infty)$. Then there exists a unique quadratic-additive mapping $F: X \rightarrow Y$ such that

$$
\|f(x)-F(x)\| \leq \begin{cases}\frac{3 \theta}{2^{p}-4}\|x\|^{p} & \text { if } p>2, \\ \frac{3 \theta}{2-2^{p}}\|x\|^{p} & \text { if } 0<p<1\end{cases}
$$

for all $x \in X \backslash\{0\}$. Moreover if $p<0$, then $f$ is itself a quadratic-additive mapping.

Proof. It follows from Theorem 2.3, Theorem 2.4, Theorem 2.5, and Remark 2.6 by putting

$$
\varphi(x, y, z):=\theta\left(\|x\|^{p}+\|y\|^{p}+\|z\|^{p}\right)
$$

for all $x, y, z \in X \backslash\{0\}$ with $L=2^{p-1}<1$ if $p<1, L=2^{2-p}<1$ if $p>2$, and $L^{\prime}=2^{-p}<1$ if $p>0$.

Corollary 3.4. Let $X$ be a normd space. Suppose that the mapping $f: X \rightarrow Y$ satisfies the inequality

$$
\|D f(x, y, z)\| \leq \theta\|x\|^{p}\|y\|^{q}\|z\|^{r}
$$

for all $x, y, z \in X \backslash\{0\}$, where $\theta \geq 0$ and $p+q+r \in(-\infty, 0) \cup(0,1) \cup$ $(2, \infty)$. Then there exists a unique quadratic-additive mapping $F: X \rightarrow$ $Y$ such that

$$
\|f(x)-F(x)\| \leq \begin{cases}\frac{\theta\|x\|^{p+q+r}}{2^{p+q+r}-4} & \text { if } p+q+r>2, \\ \frac{\theta\|x\|^{p+q+r}}{2-2^{p+q+r}} & \text { if } 0<p+q+r<1\end{cases}
$$


for all $x \in X \backslash\{0\}$. Moreover if $p+q+r<0$, then $f$ is itself a quadraticadditive mapping.

Proof. It follows from Theorem 2.3, Theorem 2.4, Theorem 2.5, and Remark 2.6 by putting

$$
\varphi(x, y, z):=\theta\|x\|^{p}\|y\|^{q}\|z\|^{r}
$$

for all $x, y, z \in X \backslash\{0\}$ with $L=2^{p+q+r-1}<1$ if $p+q+r<1, L=$ $2^{2-p-q-r}<1$ if $p+q+r>2$, and $L^{\prime}=2^{-p-q-r}<1$ if $p+q+r>0$.

\section{References}

[1] T. Aoki, On the stability of the linear transformation in Banach spaces, J. Math. Soc. Japan. 2 (1950), 64-66.

[2] L. Cădariu and V. Radu, Fixed points and the stability of Jensen's functional equation, J. Math. Soc. Japan.J. Inequal. Pure Appl. Math. 4(1) (2003), Art. 4

[3] L. Cădariu and V. Radu, Fixed points and the stability of quadratic functional equations, An. Univ. Timisoara Ser. Mat.-Inform. 41 (2003), 25-48.

[4] L. Cădariu and V. Radu, On the stability of the Cauchy functional equation: a fixed point approach in Iteration Theory, Grazer Mathematische Berichte, KarlFranzens-Universitäet, Graz, Graz, Austria 346 (2004), 43-52.

[5] P. Găvruta, A generalization of the Hyers-Ulam-Rassias stability of approximately additive mappings, J. Math. Anal. Appl. 184 (1994), 431-436.

[6] D. H. Hyers, On the stability of the linear functional equation, Proc. Natl. Acad. Sci. USA 27 (1941), 222-224.

[7] K.-W. Jun and Y .-H. Lee, A generalization of the Hyers-Ulam-Rassias stability of the pexiderized quadratic equations, II, Kyungpook Math. J. 47 (2007), 91-103.

[8] S.-M. Jung, On the Hyers-Ulam stability of the functional equations that have the quadratic property, J. Math. Anal. Appl. 222 (1998), 126-137.

[9] G.-H. Kim, On the stability of functional equations with square-symmetric operation, Math. Inequal. Appl. 4 (2001), 257-266.

[10] H.-M. Kim, On the stability problem for a mixed type of quartic and quadratic functional equation, J. Math. Anal. Appl. 324 (2006), 358-372.

[11] Y.-H. Lee, On the stability of the monomial functional equation, Bull. Korean Math. Soc. 45 (2008), 397-403.

[12] Y. H. Lee and K. W. Jun, A generalization of the Hyers-Ulam-Rassias stability of Jensen's equation, J. Math. Anal. Appl. 238 (1999), 305-315.

[13] Y. H. Lee and K. W. Jun, A generalization of the Hyers-Ulam-Rassias stability of Pexider equation, J. Math. Anal. Appl. 246 (2000), 627-638.

[14] Y. H. Lee and K. W. Jun, On the stability of approximately additive mappings, Proc. Amer. Math. Soc. 128 (2000), 1361-1369.

[15] B. Margolis and J. B. Diaz, A fixed point theorem of the alternative for contractions on a generalized complete metric space, Proc. Amer. Math. SocBull. Amer. Math. Soc. 74 (1968), 305-309.

[16] J. M. Rassias, On the Ulam stability of mixed type mappings on restricted domains Journal of Mathematical Analysis and Applications, J. Math. Anal. Appl. 276 (2002), 747-762. 
[17] Th. M. Rassias, On the stability of the linear mapping in Banach spaces, Proc. Amer. Math. Soc. 72 (1978), 297-300.

[18] I.A. Rus, Principles and applications of fixed point theory, Ed. Dacia, ClujNapoca (1979)(in Romanian)

[19] S.M. Ulam, A collection of mathematical problems, Interscience, New York, 1968, p. 63

Sun Sook Jin

Department of Mathematics Education, Gongju National University of Education,

Gongju 314-711, Korea.

E-mail: ssjin@gjue.ac.kr

Yang-Hi Lee

Department of Mathematics Education, Gongju National University of Education,

Gongju 314-711, Korea.

E-mail: yanghi2@hanmail.net 